\title{
Analysis of Seed Sorting Process by Estimation of Seed Motion Trajectories
}

\author{
Ole Thomsen Buus ${ }^{1}$, Johannes Ravn Jørgensen ${ }^{1}$, and Jens Michael Carstensen ${ }^{2}$ \\ 1 Aarhus University, Faculty of Agricultural Sciences, Department of Genetics and \\ Biotechnology, 4200 Slagelse, Denmark \\ \{0le.Buus, Johannes. Jorgensen\}@agrsci.dk \\ 2 Informatics and Mathematical Modelling, Technical University of Denmark, \\ Building 321, DK-2800 Lyngby, Denmark \\ jmc@imm.dtu.dk
}

\begin{abstract}
Seed sorting is a mechanical process in which the goal is to achieve a high level of purity and quality in the final product. Prediction and control of such processes are generally considered very difficult. One possible solution is a systems identification approach in which the seeds and their movement are directly observed and data about important process parameters extracted. Image analysis was used to extract such data from the internal sorting process in one particular seed sorting device - the so-called "indented cylinder". Twenty high speed image sequences were recorded of the indented cylinder in action, sorting a batch of barley with both whole and broken kernels. The motion trajectories and angle of escape for each seed in each frame were estimated. Motion trajectories and frequency distributions for the angle of escape are shown for different velocities and pocket sizes. A possible linear relationship is shown to exist between velocity and the angle. The temporal stability of certain parameters in the sorting process were also analysed and is shown to be quite stable for lower velocities.
\end{abstract}

Keywords: Seed sorting, indented cylinder, system identification, motion trajectories, image analysis.

\section{Introduction}

When seeds are harvested from fields they contain a number of larger impurities (for example stones, leaves, branches, insects) that need to be removed. When these impurities have been removed using various preprocessing machinery all that remains are particles of generally the same size. If necessary this relatively clean seed material can now be processed further. This later step is known as seed sorting and is the industrial application that we have focused on in this work. In seed sorting the task is to further sort or divide the preprocessed seed material into at least two individual sets of particles.

Different types of seed sorting machines are used in the industry today. By physically manipulating the seed material in a way that takes advantage of various individual physical distinguishing characteristics of the seeds (usually one 
for each type of machine) these machines are able to sort the material. Typical examples of such characteristics are (1) mass (density), (2) surface texture (friction with surfaces), (3) length, (4) general size, and (5) shape (for example round, prolonged, egg-shaped, flat).

The prediction and control of the process in these machines is generally considered very difficult. This is primarily due to the biological variations in the physical characteristics of the individual seeds. This is where the application of image analysis becomes relevant. There is a need for a systems identification approach wherein (parts of) the mechanical particle manipulation process is directly observed and useful information about important process parameters extracted. Image analysis is a natural tool for this.

In this paper we show results from experiments wherein image analysis was used to extract information from the internal process in the indented cylinder (laboratory scale). The indented cylinder is a length sorter that divides the incoming seed material into two subsets: (1) long and (2) short seeds. This is necessary for some seed species for which sorting based on other characteristics is not possible. The machine consists of a rotating cylinder lying down. The seed material is fed from one side. The inner surface of this cylinder is equipped with small pockets (indented into the metal). Due to the rotation of the cylinder these pockets carry the seeds up to a certain angle. This angle is dependent on the individual length of the seeds. In principle: Shorter seeds will be carried further than longer seeds. A catch-pan mounted at the centre of the cylinder will catch the shorter seeds while the longer seeds fall down to the bottom of the cylinder again. Due to a small tilt of the cylinder itself the longer seed material advances to the other side due to gravity. The shorter material is advanced using vibration of the catch-pan. Most of these basic principles are also depicted graphically in Figure 1(a)

Berlage et al. 234 are some of the earliest examples on the use of image analysis to analyse and improve seed sorting using prototypical machines (not indented cylinders). Dell'Aquila [7] is an example of a recent review on the subject of automated inspection of seed sorting for quality testing. On the modelling aspect again early work by Berlage et al. [1] and Churchill et al. 6] are mentionable. The analysis, modeling and simulation of the flow of particles in rotating cylinders has been done to great extent. But mostly for use in chemical, pharmaceutical, and matallurgical industries. Positron emission particle tracking (PEPT) 1011] and particle image velocimetry (PIV) 5] are examples of specialised technologies which have been applied for various purposes. Sandidi et al. [12 used a CCD camera for analysing the flow in a rotating drum used for coating tablets. Lastly we mention the summarised work in Grochowich [8] - one of the earliest full analyses of various types of seed sorting machines and their inherent complexity.

In this work we have specifically focused on deducing the angle of escape $\theta$ (with horizontal) of the individual seeds from recordings of the indented cylinder during an actual sorting process. The distribution of this important process 


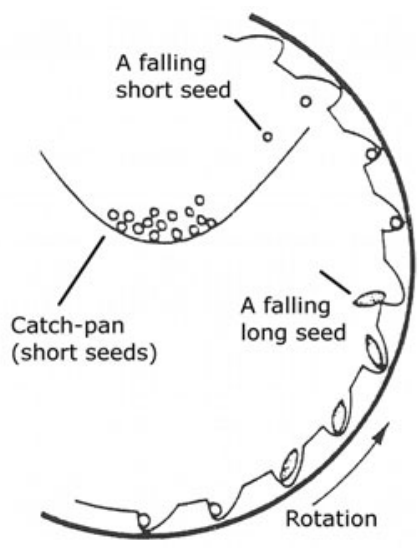

(a)

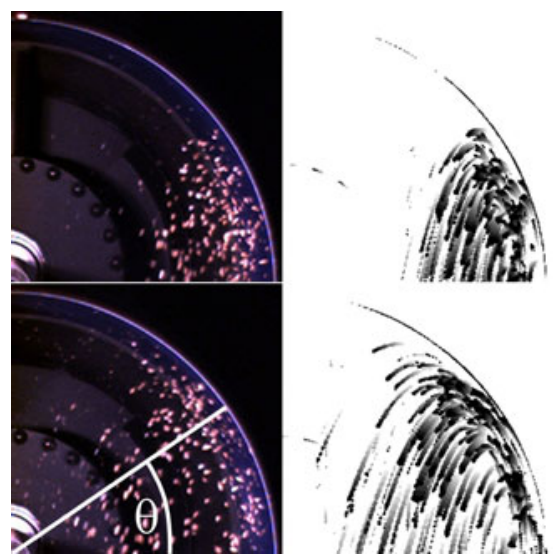

(b)

Fig. 1. A graphic and four examples of frames from the imaging data. Figure 1(a) The basic principles of the sorting process in the indented cylinder (modified from 9]). Figure 1(b). Four examples on frames acquired in the experiments. The two rows each show a situation from two different angular velocities of the cylinder (top: $26 \mathrm{rpm}$ [revolutions per minute], bottom: $34 \mathrm{rpm}$ ). To the left we see the original version and to the right we see examples on accumulated seed segmentations (accumulated of over 10 frames starting from the one on the left). The angle of escape $\theta$ is shown graphically in the lower left original version.

parameter over time says something about the machines current ability to process and sort the material given to it.

We placed a colour CCD camera in front of the active cylinder (it was in motion with material in it) with the catch-pan removed. Each image frame (taken at 260 frames/sec) was then segmented and the (approximate) location of each seed (or particle) in the image plane was extracted. Using a combination of these locations and the laws of motion (no drag included) we were able to deduce the most likely parabolic escape trajectories and thus also an estimate for the angle of escape $\theta$ for each particle. Figure 1(b) shows two examples of the images recorded for two different rotational speeds of the cylinder.

Section 2 describes in more detail the experiments, seed material (barley), and the acquired data (20 image sequences). Section 3 explains the basic methods used for extracting the seed locations and for estimating the angle of escape $\theta$. Section 4 presents the results and Section 5 concludes.

\section{Materials and Data}

\subsection{Experimental Setup}

We used a laboratory-sized indented cylinder (Westrup L-AT LAT-0801) that supports cylinders with a radius of $200 \mathrm{~mm}$ and a depth of $500 \mathrm{~mm}$. The cylinder 
had a (fixed) inclination with horizontal of $0.7^{\circ}$. A CCD colour camera (Point Grey Grasshopper GRAS-03K2C-C) was placed in front of the cylinder with the direction of sight aligned with the plane containing the cylinder's axis of rotation. The end of the cylinder closest to the camera was slightly lower due to the small inclination angle.

It was placed at a distance such that the width of the image corresponded to the apparent width of the cylinder. The monofocal lens used had a focal length of $25 \mathrm{~mm}$ and a horizontal and (deduced) vertical angle of view of $10.97^{\circ}$ and $8.24^{\circ}$, respectively. The camera was also rotated $180^{\circ}$ to use sub-sampling of the sensor lines and achieve a higher framerate. Using these parameters, the correct camera-to-cylinder distance was estimated to be $2.7 \mathrm{~m}$.

Illumination was provided by a $150 \mathrm{~W}$ halogen modelling light (SOLO 1600 $B$ ) placed between camera and cylinder. The angle of light was such that all the seed material inside the cylinder received the same amount of illumination. Note that the catch-pan was removed to give room for visual inspection with the camera.

\subsection{The Seed Material}

The seed material used was barley (Hordeum vulgare L.). The indented cylinder is particularly suitable for filtering broken (usually half) non-useful barley kernels from whole useful barley kernels.

The barley used was filtered manually using the indented cylinder. This allowed us to create a modelled seed batch with a known per particle distribution of whole and broken kernels. The modelled batch consisted of $50 \%$ whole and $50 \%$ broken barley kernels. The mixed batch had a total mass of $2 \mathrm{~kg}$ which was more than enough for the experiments.

\subsection{Experiments and Acquired Data}

The cylinder was fed with the modelled seed batch and configured to run with different settings of two important system variables. These were: (1) the cylinder rotational speed and (2) the diameter of the pockets in the cylinder. We recorded the sorting process of the cylinder for ten different rotational speeds and for two different pocket diameters. The speed was sampled in the range from $26 \mathrm{rpm}$ (revolutions per minute) to $49 \mathrm{rpm}$ with an average step of $2.56 \mathrm{rpm}$ (corresponding to angular frequencies of $2.73 \mathrm{rad} / \mathrm{s}$ to $5.14 \mathrm{rad} / \mathrm{s}$ with an average step of $0.27 \mathrm{rad} / \mathrm{s}$ ). We used two different cylinders with pocket diameters of $6.0 \mathrm{~mm}$ and $7.0 \mathrm{~mm}$.

A total of $2 \times 10=20$ image sequences were recorded at approximately 260 frames/sec for a total of ten seconds. After recording all sequences were temporarily synchronized such that the position of the cylinder circumference was the same in the first frame. This resulted in five seconds (1300 frames) of useful data in all 20 sequences (26000 frames in total). Each frame is of active dimensions $240 \times 240$ pixels and contains the upper right quadrant of the imaged cylinder circumference. 
Let $K=1300$ be the number of frames in each sequence, $V=10$ the number of velocity steps, and $D=2$ the number of pocket diameters. One can then define integer indexes $k=1, \ldots, K, v=1, \ldots, V$, and $d=1, \ldots, D$ (where $d=1$ and $d=2$ corresponds to pocket diameter $6.0 \mathrm{~mm}$ and $7.0 \mathrm{~mm}$, respectively). Any frame in any of the 20 sequences can now be described as a matrix $\mathbf{F}_{(v, d)}^{(k)} \in$ $\mathbb{R}^{N \times N}$, where $N=240$. Each sequence has also been given a mathematical name: $\mathcal{S}_{(v, d)} \in \mathbb{R}^{N \times N \times K}$. Finally, the following shorthand notations are defined: $\mathbf{F}^{(k)}$ is the $k^{\prime}$ th frame in any sequence $\mathcal{S}_{(v, d)}$ and $\mathbf{F}$ is any frame in any sequence. One final sequence exists: $\mathcal{B}$. This is a background sequence with no seeds in the scene - just the empty cylinder $(d=2)$ rotating at velocity step $v=5$.

\section{Methods}

\subsection{Estimation of Seed Locations}

Figure 2 shows the 11 image processing steps used to extract the locations of the seeds in each frame $\mathbf{F}_{(v, d)}^{(k)}$. The average $\mathbf{B}_{\text {ref }}$ of sequence $\mathcal{B}$ is the static background frame mentioned in step 5. This is used for background subtraction to acquire a global segmentation and to remove certain problematic areas of the image. The resulting absolute difference map was scaled to range $[0,1]$ and

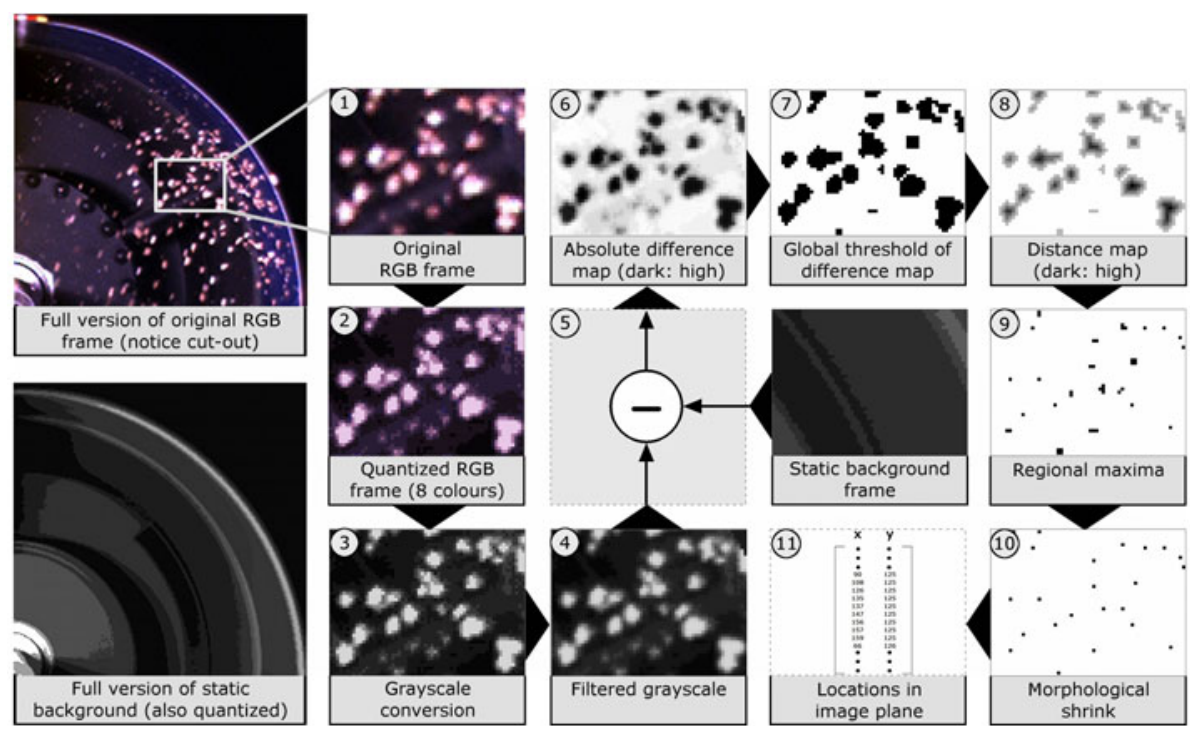

Fig. 2. The 11 image processing steps used to extract the seed locations (in the image plane). In the top-left part an example of a full frame is seen (cylinder velocity is 36 rpm [revolutions per minute]). Step 10 mentions the process of "morphological erosion". It is a continued erosion that shrinks objects without holes to single points (holes in objects are removed prior to the operation). 
a global threshold (step 7) of 0.4 was used (everything above that level was considered part of foreground/seed). Various morphological tools are then used to produce binary map containing approximate seed locations in the image plane. Finally these locations in the image plane are transformed to a 2-dimensional world space in $\mathbb{R}^{2}$ by a linear interpolation.

\subsection{Estimating the Angle of Escape $\theta$}

We have estimated the angle of escape $\theta$ and calculated the corresponding parabolic trajectory for all twenty configurations of the indented cylinder. Figure 3 show six such "trajectory plots" for rotational speeds $v=1,2,5$, 6,9 , and 10; and for both pocket sizes $d=1$ and 2 . Note the visible difference in trajectories due to change in speed. The points shown are the ones extracted from the recorded frames using the methods described above.

The estimation of $\theta$ was done for all points extracted from all frames $\mathbf{F}$ using the processing steps just described (one estimate per point). To minimize data representation complexity, the seed locations from every 10 frames were combined into a single set of points. That is, for any sequence $\mathcal{S}_{(v, d)}$ we generate $L=K / 10=1300 / 10=130$ accumulated point sets $A^{(l)}$ containing $I^{(l)}$ points $\left(x_{i}, y_{i}\right) \in \mathbb{R}^{2}$. These accumulated point sets are then analysed for $l=1, \ldots, L$ resulting in a solution set $\Theta^{(l)}$ containing $I^{(l)}$ scalars $\theta_{i} \in \mathbb{R}$.

The specific number of points $I_{(v, d)}^{(l)}$ available in each $A_{(v, d)}^{(l)}$ varies only slightly over $l$ but expectedly varies more over index $v$ and $d$. Especially for $d$ since the $1 \mathrm{~mm}$ difference in pocket diameter has the effect that different amounts of seeds are caught by the pockets.

During sorting, a seed or particle moves with the cylinder up to a certain angle $\theta$. This angle is dependent on the radius $r$ and current angular frequency $\omega$ of the indented cylinder. We model the movement of each such particle as a point $\left(x_{i}, y_{i}\right)$ moving in $\mathbb{R}^{2}$ in its own local time domain, starting at time $t_{i}=0$ when a force equilibrium (explained in great detail in Grochowich [8, Chp. 7]) accelerates the particle off the cylinder wall and (in our model, influenced now only by gravity) into a parabolic trajectory.

At that time the particle modelled as the point $\left(x_{i}, y_{i}\right)$ will have the following initial velocity and position components:

$$
\begin{aligned}
\dot{x}_{0}(\theta, \omega, r) & =-r \omega \sin \theta \\
\dot{y}_{0}(\theta, \omega, r) & =r \omega \cos \theta \\
x_{0}(\theta, r) & =r \cos \theta \\
y_{0}(\theta, r) & =r \sin \theta .
\end{aligned}
$$

The parabolic time-dependent motion of a single particle $\left(x_{i}, y_{i}\right)$ is described by components:

$$
\begin{aligned}
& x_{i}(t, \theta, \omega, r)=\dot{x}_{0}(\theta, \omega, r) t_{i}+x_{0}(\theta, r) \\
& y_{i}(t, \theta, \omega, r)=-(g / 2) t_{i}{ }^{2}+\dot{y}_{0}(\theta, \omega, r) t_{i}+y_{0}(\theta, r),
\end{aligned}
$$




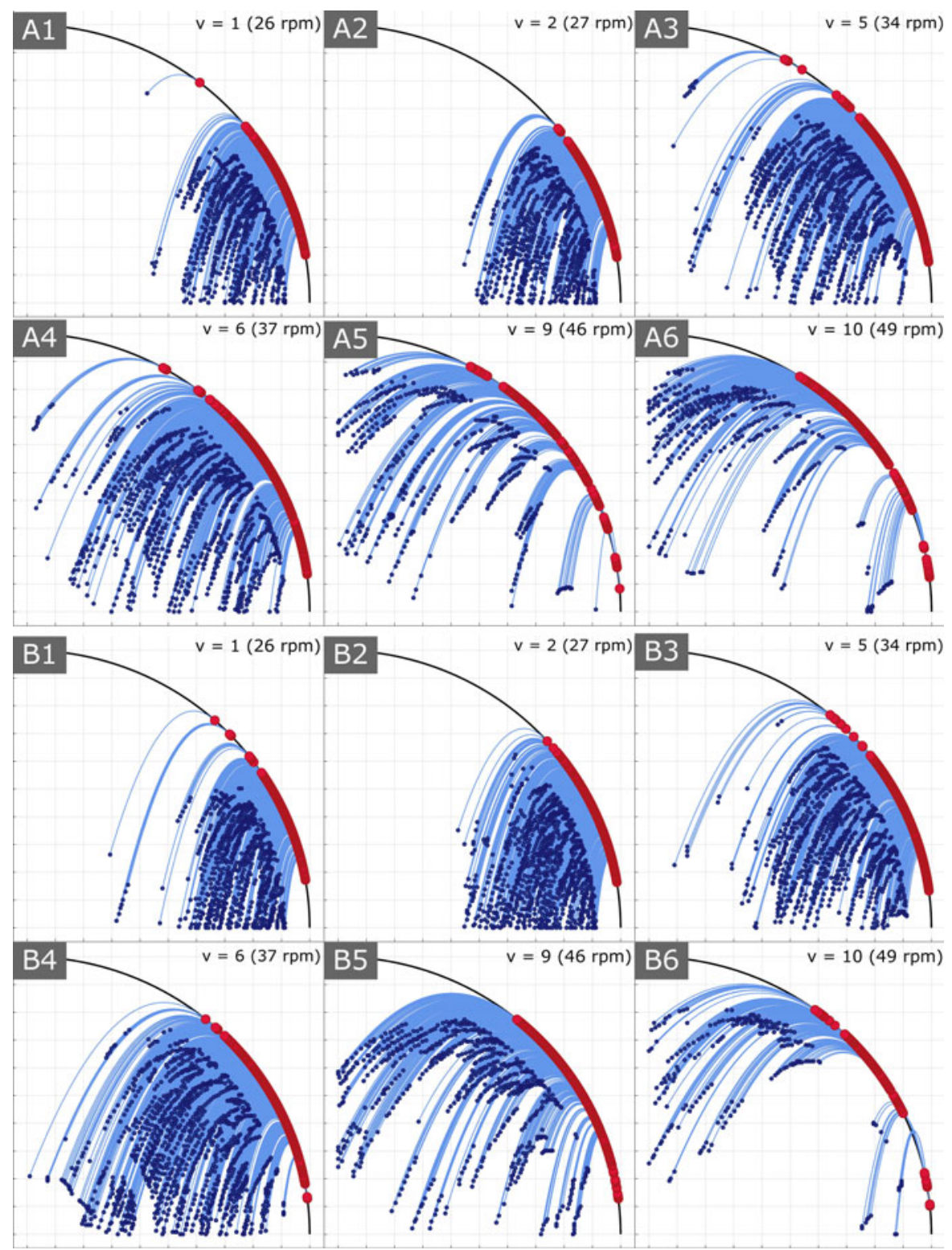

Fig. 3. Twelve "trajectory plots": In the top part (markings "A1" to "A6") they are shown for six velocities $v=1,2,5,6,9$, and 10 ; for pocket size $\mathrm{d}=1(6.0 \mathrm{~mm})$. In the lower part (markings "B1" to "B6") they are shown for the same velocities but for pocket size $d=2(7.0 \mathrm{~mm})$. The points shown correspond to the extracted point locations in $\mathbb{R}^{2}$. The markings on the circumference depict the estimated $\theta$ values. 


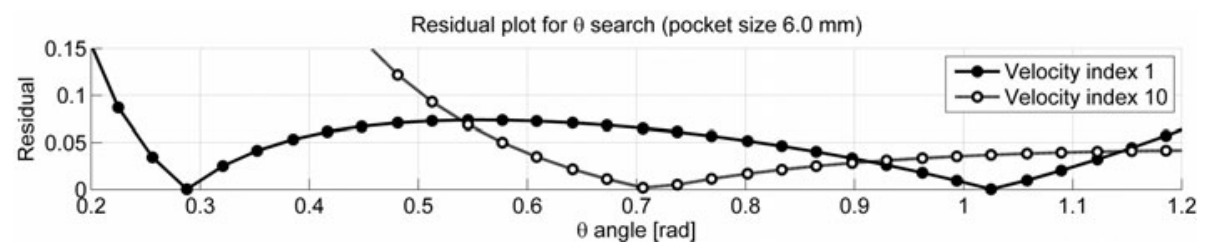

Fig. 4. Two residual curves for two distinct points: $\left(x_{100}, y_{100}\right)$ from $A_{(1,1)}^{(65)}$ and $\left(x_{160}, y_{160}\right)$ from $A_{(10,1)}^{(65)}$. That is, for both the slowest rotational speed $v=1$ ("Velocity index 1") and the fastest $v=10$ ("Velocity index 10"), though only for one pocket diameter $d=1$. On the curve for $v=1$ there are two minima at (1) $\theta=0.29 \mathrm{rad}$ $\left[16.49^{\circ}\right]$ and $(2) \theta=1.03 \mathrm{rad}\left[58.83^{\circ}\right]$. On the other curve $(v=10)$ there is only one minima at $\theta=0.71 \mathrm{rad}\left[40.90^{\circ}\right]$.

where $g=9.82 \mathrm{~m} / \mathrm{s}^{2}$ is the gravity acceleration constant. Solving for $t$ in (2a) and substituting into (2b) we get the following function (dropping the point index $i$ for generality):

$$
\tilde{y}_{\omega, r}(\theta, x)=G_{\omega} \frac{1}{r^{2}} \csc ^{2} \theta[r \cos \theta-x]^{2}+\cot \theta[r \cos \theta-x]+r \sin \theta,
$$

where the constant $G_{\omega}=-(g / 2) \omega^{-2}$ is the only factor involving gravity acceleration constant $g$ and $\omega$. The problem is now to solve (3) for $\theta$ for each point $\left(x_{i}, y_{i}\right)$. This was done numerically. First we defined a residual function:

$$
e_{\omega, r}\left(\theta, x_{i}, y_{i}\right)=\left|\tilde{y}_{\omega, r}\left(\theta, x_{i}\right)-y_{i}\right|
$$

which naturally makes it a minimization problem in $\mathbb{R}$ :

$$
\theta_{i}=\underset{\theta}{\arg \min } e_{\omega, r}\left(\theta, x_{i}, y_{i}\right)
$$

We chose to do a full numerical search in the entire range from 0 to $\pi / 2$ with a step size of $h=10^{-3} \pi / 2$ (1000 divisions). No stopping criteria was used, resulting in a list of 1000 residual values for each point $\left(x_{i}, y_{i}\right)$ to analyse further.

Figure 4 shows two plots for the residual value calculated for two points for the slowest and fastest rotational speed (see figure caption for details). When looking at the residual curve for $v=1$, there are two solutions for the angle $\theta$ (two minima exist). This is a mathematical detail easily dealt with. For current point $(x, y)$ only $\theta$ values for which the following is true can possibly be member of the solution set $\Theta_{l}: \theta<\arccos (x / r)$, where $x \leq r$. In the current example we have $x=0.17$, meaning that the upper $\theta$ limit is $\arccos (0.17 / 0.2)=0.56 \mathrm{rad}$ $\left[31.90^{\circ}\right]$. Thus only the lower $\theta$ value in the current example would be considered. Finally, we notice that for the residual from motion at velocity step $v=10$ there is only one minima at a higher angle than for the $v=1$ curve. 


\section{Results and Discussion}

The methods presented in Section 3 made it possible to analyse for what angle with horizontal the seeds are thrown off the cylinder wall. For each solution set $\Theta_{l}$ available at each time index $l$ in any sequence, we created a frequency distribution $h_{l}$ over $\theta$ and estimate a normal fit with parameters $\mu_{l}$ and $\sigma_{l}$. Beyond this, all distributions $h_{l}$ for each sequence were also summed and a normal density fitted as well. This made it possible to statistically describe the behaviour of $\theta$ for each full sequence using only two parameters (we refer to it specifically as the summed distribution).

In Figure 5 we see examples of the summed frequency distributions with the corresponding normal fit superimposed. They are specifically shown for three

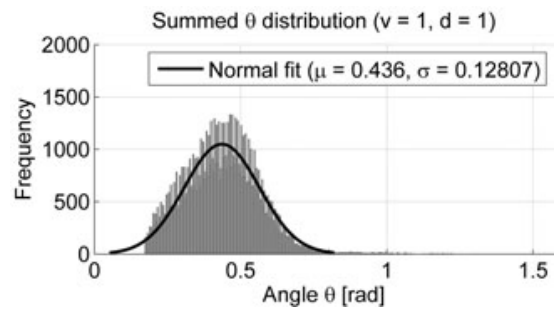

(a)

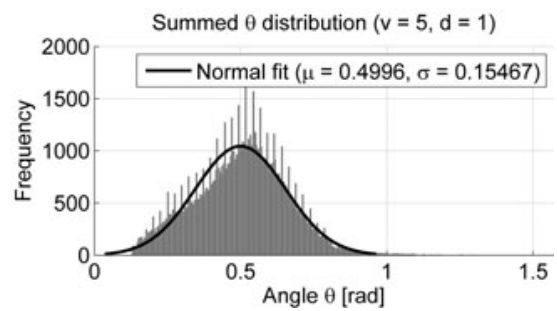

(c)

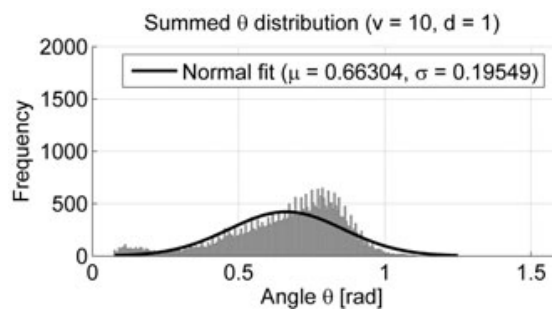

(e)

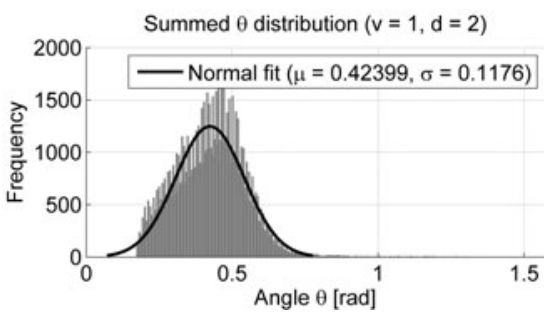

(b)

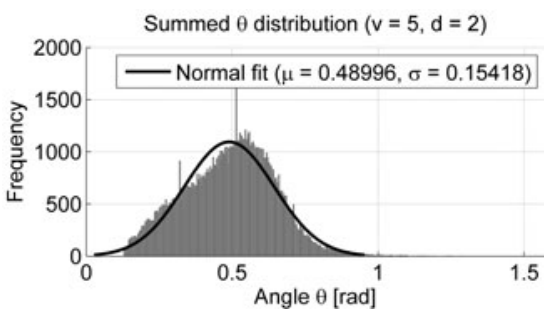

(d)

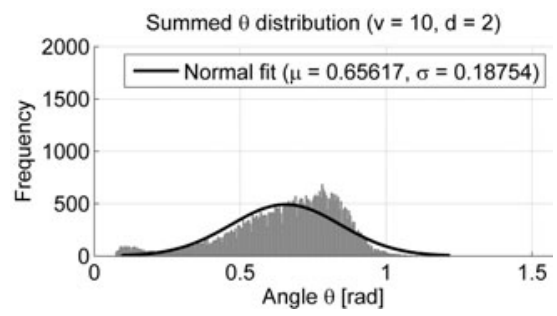

(f)

Fig. 5. Six $\theta$ frequency distributions with fitted normal density superimposed. Fig. $5(\mathrm{a})$ $5(\mathrm{c})$ and, 5(e) show the distributions and normal fit for velocities $v=1,5$, and 10; for pocket diameter $d=1(6.0 \mathrm{~mm})$. Fig. $5(\mathrm{~b})$ 5(d) and, $5(\mathrm{f})$ show the distributions and normal fit for the same three velocities, but now instead for pocket diameter $d=2$ $(7.0 \mathrm{~mm})$. 


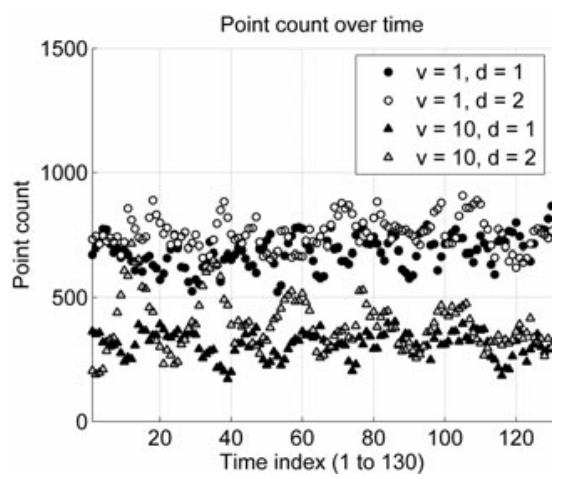

(a)

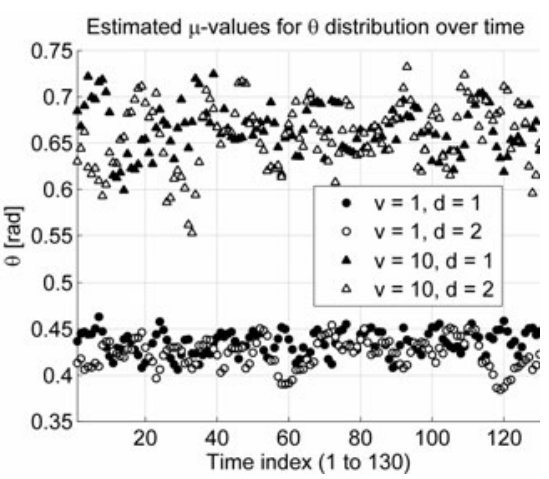

(b)

Fig. 6. Two scatter plots showing different variables gathered over time: Figure 6(a) shows a scatter plot of point counts $I_{(d, v)}^{(l)}$ over $l, \ldots, L$ for $v=1,10$ and $d=1,2$. In other words: The number of points accumulated for every ten frames, for the slowest and fastest velocity and for both pocket sizes. Figure 6(b) shows a scatter plot of estimated normal $\mu$-parameter from frequency distributions generated from solution sets $\Theta_{l}$ over the same index ranges as in Figure 6(a)

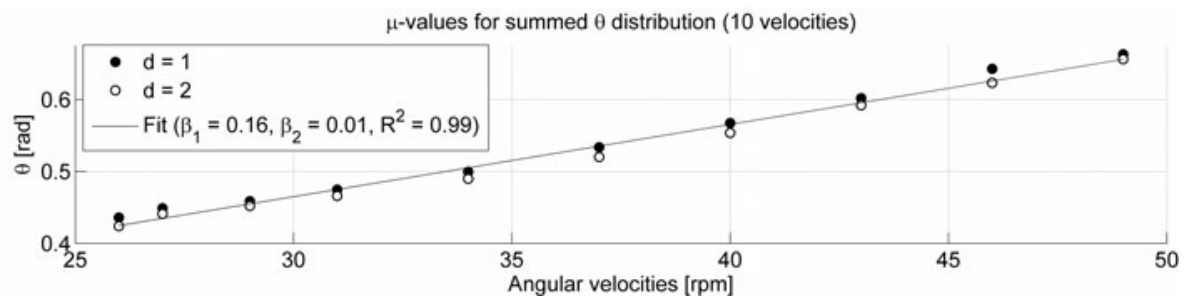

Fig. 7. Scatter plot and a linear regression fit of the $\mu$-parameter estimated from the summed (accumulated over time) frequency distributions under the assumption that the distribution is normal. The data are shown for all ten angular velocities and for both pocket sizes. The linear fit was done using all 20 data points (with rpm values as predictors).

velocity steps: (1) slowest, middle $(d=5)$, and fastest; for both pocket sizes. Not much difference in appearance of the distribution over pocket sizes can be observed directly - mostly the difference is within changes in rotational speed. Generally, for a higher speed there seems to be a higher probability for larger values of $\theta$. Also, for the highest speed the distribution also seems to become slightly multi-modal. Two modes are observable in figures $5(\mathrm{e})$ and $5(\mathrm{f})$ and also in the trajectories plotted in Figure 3 (for that particular rotational speed).

Figure 6(a) show a scatter plot of number of points available in each time step $l$. The values are shown for both slowest and fastest velocity step, as well as for both pocket sizes. The system seems to have some stability over time. It is also evident that for the fastest velocity step, fewer seeds are extracted than for the slowest velocity step. Figure 6(b) show for the same velocity steps and pocket 
sizes the estimated $\mu_{l}$ parameter for the frequency distributions generated over time. Again we observe stability. On top of this, we observe that $\theta$ is generally larger for the fastest velocity step than for the slowest velocity step. Also, the number of points extracted is fewer for the fastest velocity step than for the slowest.

Figure 7 show the estimated $\mu$-parameter for the summed frequency distributions over all ten rotational speeds and for both pocket sizes. The regression fit indicates the possibility of a linear relationship between the rotational speed of the indented cylinder and the angle of escape $\theta$.

\section{Conclusions}

In this work we have experimentally verified a certain behaviour of the sorting process in the indented cylinder. First and foremost, at least for the 5 seconds of recording that we have dealt with, we show that the process has some stability (but mostly for the lower velocity steps). Secondly, we show a linear relation between rotational speed and angle of escape $\theta$. As mentioned in the introduction there is a need for a systems identification approach in where the indented cylinder is analysed to acquire information about important parameters. Thus a third results is a more tentative one: We have shown that image analysis can be used for flow analysis of particles moving in an indented cylinder. This is a novel step toward the goal of predicting and controlling the sorting process in these machines.

One final important remark: Our choice of using a seed batch with $50 \%$ whole and $50 \%$ broken barley kernels will undoubtedly have had an impact on the distribution of the angle of escape $\theta$. Had the kernel size distribution been more realistic, for instance, with $10 \%$ broken, and $90 \%$ whole or opposite, then we would likely have observed a multi-modal frequency destribution of $\theta$. That would be interesting to try in the future.

\section{Acknowledgements}

We would very much like to acknowledge the support and guidance received from Westrup A/S, Denmark.

\section{References}

1. Berlage, A.G., Bilsland, D.M., Brandenburg, N.R., Cooper, T.M.: Experimental indent cylinder for separating seeds. Transactions of the American Society of Agricultural Engineers 27(2), 358-361 (1984)

2. Berlage, A.G., Churchill, D.B., Cooper, T.M., Bisland, D.M.: The application of new technologies to seed conditioning. Journal of Agricultural Engineering Research 42(3), 193-202 (1989)

3. Berlage, A.G., Cooper, T.M., Aristazabal, J.F.: Machine vision identification of diploid and tetraploid ryegrass seed. Transactions of the American Society of Agricultural Engineers 31(1), 24-27 (1988) 
4. Berlage, A.G., Cooper, T.M., Carone, R.A.: Seed sorting by machine vision. Agricultural Engineering 65(10), 14-17 (1984)

5. Buchhave, P.: Particle image velocimetry-status and trends. Experimental Thermal and Fluid Science 5(5), 586 (1992), special Issue on Experimental Methods in Thermal and Fluid Science

6. Churchill, D.B., Berlage, A.G., Bilsland, D.M., Cooper, T.M.: Decision-support system development for conditioning seeds with indent cylinder. Transactions of the American Society of Agricultural Engineers 32(4), 1395-1398 (1989)

7. Dell'Aquila, A.: Towards new computer imaging techniques applied to seed quality testing and sorting. Seed Science and Technology 35(3), 519-538 (2007)

8. Grochowicz, J.: Machines For Cleaning And Sorting Of Seeds. Department of Agriculture (1980) (translated from polish)

9. Lampeter, W.: Die Saatgutaufbereitung. VEB Deutscher Landwirtschaftsverlag, Berlin (1965)

10. Parker, D.J., Dijkstra, A.E., Martin, T.W., Seville, J.P.K.: Positron emission particle tracking studies of spherical particle motion in rotating drums. Chemical Engineering Science 52(13), 2011-2022 (1997)

11. Pianko-Oprych, P., Nienow, A., Barigou, M.: Positron emission particle tracking (pept) compared to particle image velocimetry (piv) for studying the flow generated by a pitched-blade turbine in single phase and multi-phase systems. Chemical Engineering Science 64(23), 4955 - 4968 (2009)

12. Sandadi, S., Pandey, P., Turton, R.: In situ, near real-time acquisition of particle motion in rotating pan coating equipment using imaging techniques. Chemical Engineering Science 59(24), 5807-5817 (2004) 